\title{
La grande fabbrica fordista. Culture politiche e scienze sociali alla prova del neocapitalismo
}

La grande usine fordiste. Cultures politiques et sciences sociales à l'égard du néocapitalisme

Fordist Factories. Political Cultures and Social Sciences facing Neocapitalism

Bruno Settis

\section{OpenEdition}

Journals

Edizione digitale

URL: http://journals.openedition.org/cei/2965

DOI: 10.4000/cei.2965

ISSN: 2260-779X

Editore

UGA Éditions/Université Grenoble Alpes

\section{Edizione cartacea}

Data di pubblicazione: 20 avril 2016

Paginazione: 189-202

ISBN: 978-2-84310-323-0

ISSN: 1770-9571

Notizia bibliografica digitale

Bruno Settis, «La grande fabbrica fordista. Culture politiche e scienze sociali alla prova del

neocapitalismo», Cahiers d'études italiennes [Online], 22 | 2016, online dal 01 janvier 2017, consultato il 27 mars 2021. URL: http://journals.openedition.org/cei/2965 ; DOI: https://doi.org/10.4000/cei.2965 


\title{
LA GRANDE FABBRICA FORDISTA. CULTURE POLITICHE E SCIENZE SOCIALI \\ ALLA PROVA DEL NEOCAPITALISMO
}

\author{
Bruno Settis \\ Centre d'histoire de Sciences Po et Scuola Normale di Pisa
}

Le fabbriche, per un profano, sono un po' come foreste, fitte di tronchi di ferro dei torni e delle presse, attraversate in alto dai rami delle gru, irte di un sottobosco di trucioli meccanici. A camminare in mezzo a questa vegetazione meccanica, capita in certi punti di perdere di vista l'uomo: certo ce n'è qualcuno nascosto qua o laggiù, ma a chi passa, di scorcio, si profila una foresta di sole macchine che si muovono su se stesse senza posa.

Questo profano che esplorava la Fiat Spa nel gennaio 195I era Italo Calvino, giornalista dell' «Unità» piemontese, attento e partecipe osservatore della vita e dei conflitti nella più grande industria d'Italia ${ }^{\mathrm{I}}$. Ad Alain Touraine l'ambiente della grande fabbrica appariva invece come un "raccourci de l'évolution historique»": un complesso di officine con la sua geografia economica interna, in cui il lavoro di catena e lo sviluppo dell'automazione convivevano con aree o singoli procedimenti caratterizzati dall'abilità manuale e dalla cultura professionale. Nelle officine di Torino, come in quelle di Billancourt, in certi settori si trovavano ancora lavoratori altamente specializzati, mentre in altri comparivano bracci meccanici e convogliatori aerei, a Mirafiori cominciava a svilupparsi una rete di processi automatizzati, alla Renault si cominciava ad applicare lo Statistical Process Control System. Per Serge Mallet, la Francia intera era «un musée de l'industrie», dove si potevano trovare fabbriche ad avanzata automazione e manifatture rimaste ferme all'inizio del secolo. Dirà Calvino nel

I. I. Calvino, Sezione Fiat che vai, sfruttamento che trovi. Tutti i trucchi diventano buoni per 'tagliare $i$ tempi' alla SPA, «'Unità» piemontese, 26 gennaio I95I.

2. A. Touraine, L'Évolution du travail ouvrier aux usines Renault, Parigi, CNRS, I955, p. I39. 
I960 che in Italia «abbiamo insieme a portata di mano Detroit e Calcutta, tutto ormai è mescolato assieme, Nord e Sud, tecnica avanzata e aree depresse, e le ideologie più diverse convivono, si contaminano, s'abbarbicano le une alle altre» ${ }^{3}$.

Le trasformazioni politiche, sociali ed economiche che investirono l'Europa occidentale nel secondo dopoguerra costituiscono un materiale che il mestiere di storico trova ancora difficoltà a maneggiare. Si tratta di entrare nel vivo delle sorti magnifiche e progressive di un'espansione economica passata alla memoria collettiva come 'miracolosa', ma lacerata dal riprodursi e rimodularsi delle diseguaglianze, dalle divisioni della guerra fredda, da conflitti sociali profondi, da progetti politici e culturali divergenti. Per avvicinarsi allo studio della storia del 'miracolo economico europeo' è necessario usare le lenti bifocali: sapendo guardare, cioè, in basso alle concrete vicende sui luoghi di lavoro, riconoscendone tanto le specificità quanto le caratteristiche comuni (e dunque trovare le possibilità di comparazione) e, in alto, alle politiche economiche, alle proposte politiche del movimento operaio e dei partiti delle sinistre e, infine, ai dibattiti e alle interpretazioni generali che emergevano dalle scienze sociali ${ }^{4}$.

È bene precisare subito che utilizzo qui il termine 'fordismo' nel senso 'micro' di un sistema organizzativo della produzione e delle relazioni industriali in fabbrica e non nel senso 'macro' di un regime di accumulazione o di un contratto sociale, che avrebbe caratterizzato il progresso economico e sociale dei 'Trenta Gloriosi' attorno alla spirale virtuosa di produzione di massa e consumo di massa. Questo secondo concetto è una costruzione sociologica di origine italo-francese: qua ne vediamo la genealogias.

In Italia la Fiat, in Francia la Renault, la Citroën e la Peugeot avevano assunto il fordismo come orizzonte già durante o subito dopo la prima guerra mondiale: arrivarono dunque già da protagoniste all'appuntamento del decollo economico degli anni I950, grazie ad una struttura produttiva

3. I. Calvino, Dialogo di due scrittori in crisi, da una conferenza letta nel marzo e aprile I96I in varie città della Svizzera, Svezia, Norvegia, Danimarca, ora in Una pietra sopra, Mondadori, Milano 2005 (1980), p. 8I.

4. Traggo la bella immagine delle lenti bifocali — ovviamente applicata a un diverso oggetto — da G. Melis, Storia dell'amministrazione italiana (I86I-I993), Bologna, Il Mulino, I996, p. 7.

5. Di proiezione del fordismo oltre i confini della fabbrica si parlava ovviamente, come accennerò più avanti, già dagli anni 1920; ma l'uso delle analisi di Gramsci all'interno del dibattito sulla 'fine del fordismo' e sul delinearsi del 'postfordismo' è stato spesso meccanico, e richiederebbe invece una riflessione storica più approfondita e articolata su più livelli, di cui questo scritto vorrebbe essere un tassello. Per una critica netta alla fortunata formula di J. Fourastié, cfr. C. Pessis, S. Topçu e C. Bonneuil (a cura di), Une autre histoire des «Trente Glorieuses" : modernisation, contestations et pollutions dans la France d'après-guerre, Parigi, La Découverte, 2013, concentrata sul fronte dell'impatto ecologico della crescita economica, in particolare il saggio di R. Bécot, Les germes de la préoccupation environnementale dans le mouvement syndical: sur les rapports entre syndicalisme et productivisme, pp. 23I-248. 
organizzata attorno alla catena di montaggio secondo il principio dell'integrazione verticale. Se c'è una frase che racchiude il cuore dell'intrecciarsi di battaglia politica e battaglia economica è quella, ben più di un gioco di parole, con la quale, nell'aprile 1948, Paul G. Hoffman assumeva il timone del Piano Marshall: "The real battle today is between the American assembly line and the Communist Party line» ${ }^{6}$. In Italia come in Francia, a Torino come a Billancourt, questa lotta durò decenni: in questo convegno abbiamo discusso il periodo conclusosi con l'ondata di occupazioni delle fabbriche del 1968-1969, ma si tratta di farlo evitando teleologie — che sarebbero di fatto delle scorciatoie. Quello di Hoffman era, com'è ovvio, un grido di battaglia, che voleva schernire i comunisti come nemici della prosperità e della modernizzazione, come avvoltoi che pascolavano sulla miseria della guerra. Al contrario, negli anni della ricostruzione le sinistre europee forgiarono uno spirito di energico produttivismo, spesso addirittura di lealtà all'azienda, come ha mostrato Charles Maier affiancando comunisti italiani e francesi. Con diverse oscillazioni tra collaborazione e antagonismo, in buona sostanza, i comunisti e le sinistre furono tutt'altro che ostili alle politics of productivity di stampo fordista, cui volevano semmai dare una torsione democratica: in basso con la contrattazione dei salari e dei diritti e con esperimenti di partecipazione degli operai alle scelte dell'azienda; in alto, e forse più spesso, con la ricerca di una gestione condivisa delle politiche economiche a livello di governo nazionale e locale ${ }^{7}$.

Se alla Fiat si verificò un più tradizionale e frontale scontro tra sinistre operaie e grande proprietà capitalistica, la Renault presentava caratteristiche diverse. Il controllo della Renault era stato assunto dal governo provvisorio del generale de Gaulle, dapprima come misura d'emergenza, per poi decretarne la nazionalizzazione il $\mathrm{I}^{\mathrm{o}}$ gennaio 1945 . Nominato direttore generale Pierre Lefaucheux, cambiato il nome in Régie nationale des usines Renault, prendeva forma un inedito 'fordismo di Stato' e questa colossale impresa pubblica, nazionalizzata e concorrenziale, assurgeva a pietra angolare dell'economia mista francese. Un'industria pilota' anche nel campo delle relazioni industriali, sia quando gli scioperi del 1947 avevano provocato la rottura del sindacato e la nascita di Force ouvrière con l'obiettivo, comune alla Cisl, di seguire il modello delle labor relations

6. Citato in D.E. Nye, America's Assembly Line, Cambridge, MIT Press, 2013, p. I27.

7. Cfr. C. Maier, Between Taylorism and Technocracy. European Ideologies and the Vision of Industrial Productivity in the I920s, "Journal of Contemporary History", vol. 5, $\mathrm{n}^{\circ} 2$, aprile 1970, ora in Id., In Search of Stability. Explorations in Historical Political Economy, Cambridge University Press, 1987. 
di marca americana ${ }^{8}$; sia nell'autunno 1955 quando, a seguito di un estenuante braccio di ferro, la direzione della Renault rompeva con la prassi degli accordi separati e coinvolgeva la Cgt di Benoît Frachon nei contratti che ponevano le coordinate dei rapporti con i lavoratori dei vent'anni successivi ${ }^{9}$. In tale funzione di pilota appariva come la punta avanzata di quella che i critici chiamavano l'integrazione della classe operaia' nel sistema, uno dei temi caratterizzanti della letteratura sul neocapitalismo, ovvero lo smussamento della conflittualità della classe operaia, la crescente convergenza d'interesse del lavoro e del capitale nella crescita del benessere.

Negli anni 1950 la grande fabbrica appariva come un vero bastione di efficienza e razionalità, un avamposto del capitalismo avanzato: il luogo dove il management, privato o pubblico, poteva anticipare i tempi dell'adattamento delle istituzioni alle trasformazioni tecniche ed economiche. Il centro irradiatore, insomma, della tendenza del capitale a includere tutta la società sotto la sua organizzazione, sottomettendo sia le irrazionalità del mercato che le passioni e le ragioni dei conflitti. L'elemento nuovo era, nella sintesi di Lelio Basso, la capacità «di controllare in una certa misura le contraddizioni interne, e di prevederne e in parte prevenirne le conseguenze, attenuando quindi le tensioni sociali che ne potrebbero derivare» ${ }^{10}$. Ma proprio questo era il nodo teorico dello scontro. Il movimento operaio, socialista ma anche cattolico, si divideva sulla questione se le contraddizioni del capitalismo venissero effettivamente 'superate' grazie al progresso tecnico ed al benessere, nel qual caso anche le sinistre avrebbero dovuto prendere parte alla gestione di questo superamento, incoraggiarlo, dirigere il capitalismo insieme ai capitalisti — o meglio di essi; oppure, come riteneva Basso, le contraddizioni erano solo 'controllate', nascoste sotto il tappeto, e in tal caso compito delle sinistre marxiste sarebbe stato farle riemergere e continuare a far leva su di esse.

È un concetto che possiamo chiamare, riprendendo il linguaggio dell'epoca, 'neocapitalistico', o meglio 'tecnocratico', sia presso i suoi sostenitori che presso i suoi critici (non solo di sinistra) ricordando che i due campi, per quanto avversi, non erano affatto privi di dialogo né di somiglianze o addirittura convergenze. Non è difficile individuarne la

8. F. Romero, Gli Stati Uniti e il sindacalismo europeo 1944-I95I, Roma, Edizioni Lavoro, 1989; M. Antonioli, M. Bergamaschi e F. Romero (a cura di), Le scissioni sindacali. Italia e Europa, Pisa, Biblioteca Franco Serantini, I999.

9. P. Fallachon, Les grèves de la Régie Renault en I947, «Le Mouvement Social», nº 8I, ottobre-dicembre I972, pp. III-I42; C. Sardais, La naissance d'une institution : Pierre Lefaucheux et la Régie Renault, "Revue française de gestion", vol. 6, $\mathrm{n}^{\circ} \mathrm{I} 59,2005$.

IO. L. Basso, Neocapitalismo e 'socialisti moderni', "Problemi del Socialismo", n ${ }^{\circ}$ 9, 1966, ora in Neocapitalismo e sinistra europea, Bari, Laterza, 1969, pp. 255-284. 
genealogia: nei dibattiti dei primi anni 1930 dove, per dirla in una formula frettolosa, si denunciava che la crisi del 1929 aveva segnato la fine del capitalismo liberale ed era necessario rimodellarlo sulle suggestioni del fordismo americano o del Piano sovietico. Già allora si parlava in Francia di 'néocapitalisme' e di superamento del conflitto tra capitale e lavoro ${ }^{\text {II. }}$ Negli anni 1950 il problema nodale sembrava invece che gli stessi successi del capitalismo lo avessero reso ingovernabile dalla politica: in Italia il logoramento del centrismo stentava a lasciar spazio a una nuova formula politica, in Francia pianificazione e riformismo finivano per arenarsi nelle deludenti esperienze dei governi Mendès France e Mollet e nei proverbiali pantani della Quarta Repubblica. La cultura riformista e socialista francese era stata più rapidamente ricettiva della letteratura tecnocratica e sociologica statunitense e aveva aperto il dibattito sulle trasformazioni del capitalismo prima che in Italia (dove esso cominciò ad arrivare tramite canali quali la rivista olivettiana "Comunità» e le edizioni omonime): è notevole che uno dei primi e più significativi testi, un manifesto delle politiche economiche riformiste e della piena occupazione, fosse stato scritto, come recitava la copertina, proprio "par Pierre Mendès France, Président du Conseil, et Gabriel Ardant, Commissaire général à la productivité» ${ }^{12}$. Questa ampiezza di dibattito non portò però all'uscita da quei pantani ma al contrario fu essa stessa, come sottolineava Bruno Trentin nella sua celebre analisi delle culture del neocapitalismo, un elemento della "crisi aperta e riconosciuta dell'ideologia riformista ${ }^{\mathrm{I}}{ }^{3}$.

A queste tensioni si rispose in un caso con un'apertura politica - il travagliato esperimento del centrosinistra - e nell'altro, dove i socialisti avevano già avuto modo di entrare nella 'stanza dei bottoni' e non dar buona prova di sé, con una stretta autoritaria — la 'rivoluzione dall'alto' di de Gaulle. Proprio quest'ultima, con la sua capacità di tenere insieme proposte tecnocratiche (spesso in continuità con i governi socialisti e radicali) e persino, nella sua ala sinistra, opzioni di cogestione in tinta laburista $^{14}$, si sarebbe imposta sullo scenario europeo come un campione di una destra non più (o non tanto) conservatrice, ma energicamente 'sviluppista’, dirigista, capace di mettere — meglio della Dc — l’ideologia

II. R. F. Kuisel, Capitalism and the State in Modern France. Renovation and Economic Management in the Twentieth Century, Cambridge, Cambridge University Press, I98I, e A. Salsano, Ingegneri e politici. Dalla razionalizzazione alla rivoluzione manageriale, Torino, Einaudi, 1997.

I2. P. Mendès France e G. Ardant, La science économique et l’action, Liegi, Unesco-Julliard, I954.

13. B. Trentin, Le dottrine neocapitalistiche e l'ideologia delle forze dominanti nella politica economica italiana, ora in Id., Da sfruttati a produttori. Lotte operaie e sviluppo capitalistico dal miracolo economico alla crisi, Bari, De Donato, 1977.

I4. L'Union démocratique du Travail di Louis Vallon e René Capitant (I959-I967). 
del cattolicesimo sociale al servizio dell'armonizzazione dei conflitti. E soprattutto capace di tenere - meglio dei socialisti - il polso di un capitalismo dinamico.

L'accoppiamento di autoritarismo e integrazione appariva a molti osservatori, in Francia e fuori, l'essenza sociale del gollismo. Nel 1964 Saverio Tutino, giornalista dell' «Unità», scriveva per esempio che alla Renault, con i premi e gli aumenti calcolati in base alla produttività,

[...] questo processo di 'integrazione' si andava precisando come un sottile strumento di corruzione, un metodo di assoggettamento del sindacato all'interno di ogni singola industria che alla lunga avrebbe potuto portare lontano nella liquidazione di ogni autonomia sindacale, lungo il cammino neocapitalistico ${ }^{\mathrm{IS}}$.

Non mancava però chi riteneva che il sindacato, anzi lo stesso Partito comunista fossero ormai fattori dell'integrazione, condannati ad un ruolo strumentale e complementare nella fabbrica fordista e nella società del benessere neocapitalista. È questa la visione che emerge dal Diario di un operaio, che copriva il triennio 1956-1959, di Daniel Mothé, attrezzista alla Renault e militante di Socialisme ou Barbarie. Il diario si apriva pochi mesi dopo gli accordi del 1955 e conteneva una vivida descrizione della vita in fabbrica: ma la rabbia era rivolta, più ancora che verso la direzione $\mathrm{o} i$ cronometristi, verso la disonestà dei comunisti, il loro appoggio all'occupazione di Budapest e alla guerra in Algeria, il razzismo verso gli operai algerini, l'abulia degli operai di fronte all'oppressione in fabbrica e, infine, al 'golpe' di de Gaulle. Nel 1960 Raniero Panzieri lo fece pubblicare da Einaudi, tradotto da Danilo Montaldi: fu momento non secondario del suo crescente dissidio con i colleghi della casa editrice, che di lì a poco avrebbero votato per la sua espulsione.

Nel nuovo regime la borghesia ha modernizzato le proprie istituzioni. Ha reso visibile e rafforzato il proprio potere. La classe operaia, invece, continua a conservare le sue vecchie istituzioni burocratizzate e sclerotiche, sulle quali ha sempre meno potere. $\mathrm{E}$, inversamente, queste vecchie istituzioni si integrano e sono necessarie al regime proprio perché sono inefficaci per i lavoratori ${ }^{16}$.

Una complementarità che sfociava, insomma, nella complicità: e in questa accusa Mothé (e con lui Panzieri) coinvolgeva Cgt e Cgil, Pcf e

15. S. Tutino, Gollismo e lotta operaia, Torino, Einaudi, 1964. Per una un'interpretazione di de Gaulle come modernizzatore autoritario anche L. Magri, Ipotesi sulla dinamica del gollismo, "Nuovi Argomenti», $\mathrm{n}^{\circ}$ 35-36, novembre I958-febbraio 1959; sulla figura di Magri e i suoi rapporti con la sinistra europea e soprattutto francese, vedi il bel profilo commemorativo di P. Anderson, Lucio Magri, 1932-20II, «New Left Review», $\mathrm{n}^{\circ} 72$, novembre-dicembre $201 \mathrm{I}$.

I6. D. Mothé, Diario di un operaio, 1956-1959, Torino, Einaudi, I960, trad. di D. Montaldi (ed. originale Journal d'un ouvrier, Parigi, Éditions de Minuit, 1959), p. I45. 
Pci; lo stesso dirà Serge Mallet nel 1963. Una battuta lo esprime meglio di qualunque analisi: quando Mothé scrive sul diario, in una nota del maggio 1957, che i bisticci tra i sindacati gli ricordano quelli... tra Don Camillo e Peppone ${ }^{17}$.

L'integrazione, certo, fu un mito di quegli anni, destinato a infinite smentite già prima del 1968; ma era un modo di descrivere tendenze oggettive che stavano alla base della crisi delle sinistre. Nel 1966 Lelio Basso criticò durissimamente quella parte delle sinistre europee, come i socialisti riformisti francesi e i laburisti di Wilson, che

[...] mutua[va] dal pensiero borghese l'idea che nella società capitalistica le forze di integrazione (essenzialmente democrazia politica e benessere economico) siano nettamente superiori alle forze di rottura (contraddizioni dello sviluppo, contrasti di classe) e alla sociologia marxista del conflitto tende[va] a sostituire una sociologia del consenso di ispirazione americana ${ }^{18}$.

Le novità del neocapitalismo ponevano ai marxisti nuove domande ma essi, insisteva Basso, non potevano rinunciare alla «contraddizione fondamentale» individuata da Marx: ovvero non semplicemente l'opposizione tra capitale e lavoro, bensì la dialettica tra il carattere sempre più sociale della produzione e quello privato dell'appropriazione del prodotto e del profitto.

Vediamone un esempio concreto. Per il Pci e per la Cgil di Di Vittorio il 1955 era stato l'anno, sotto lo sprone della sconfitta alle elezioni interne alla Fiat, del 'ritorno alla fabbrica'. Nello sforzo di far passare nella Cgil la svolta operaista del 1955 era impegnato in prima linea Vittorio Foa, allora deputato socialista e segretario della Fiom. Per la sua eccezionale capacità di far mettere alla politica radici nell'unione di sensibilità teorica ed esperienza della quotidianità operaia, Foa fu uno dei protagonisti di questa stagione, e le sue lucide riflessioni sono tra le più preziose per lo storico di oggi. In una conferenza del novembre 1955 Foa citava due 'autorità': Il Capitale di Marx e «un compagno dell'Alfa Romeo».

Un compagno dell'Alfa Romeo, qualche giorno fa, mi diceva una frase che credo sia presente in voi che vivete la fabbrica in tutta la sua drammaticità: «Un’ora di lavoro di ieri, di un anno fa, di due anni fa, non è più un'ora di lavoro di oggi». È vero, un'ora di lavoro è sempre un giro della lancetta dell'orologio, ma in realtà il contenuto di quell'ora di lavoro, cioè la quantità di lavoro che noi diamo in quell'ora, non è più oggi quello di ieri. Oggi, con la realizzazione tecnica più avanzata, con la disciplina delle macchine e con quella della discriminazione, quell'ora di lavoro diventa assai

I7. Ivi, p. 97.

I8. L. Basso, Neocapitalismo e 'socialisti moderni', cit., p. 260. 
più carica di contenuto lavorativo di un'ora di ieri. E noi dobbiamo discutere di quello che diamo al padrone, non ci basta discutere coi contratti nazionali o anche coi contratti aziendali quello che prendiamo in salario... ${ }^{19}$

Il divario tra la crescita galoppante della produttività e del ritmo del lavoro e quella, a singhiozzi, dei salari era la base oggettiva ${ }^{20}$ di quella che Di Vittorio chiamava la «lotta contro il supersfruttamento» e che si coniugava alla richiesta di far «entrare la Costituzione nelle fabbriche», destinata a concretizzarsi non prima del maggio 1970 con lo Statuto dei Lavoratori. Grazie ai viaggi dei dirigenti, degli ingegneri e dei sindacalisti organizzati sotto l'ombrello del Piano Marshall, Fiat e Renault furono le imprese più ricettive verso la 'seconda generazione' del fordismo, la Detroit Automation ${ }^{21}$. In questo caso con il termine 'automazione' s'intende non più solo l'organizzazione scientifica del lavoro attorno alla catena di montaggio, ma anche la pratica di sostituire progressivamente "con l'ausilio dei metodi e degli apparecchi più progrediti, e nella misura in cui lo consentono gli scopi dell'economia, la forza-lavoro umana, in tutte le sue funzioni, con macchine che vengono a loro volta controllate meccanicamente». Questa è la definizione proposta da Friedrich Pollock nel suo testo classico sull'automazione, frutto nel 1956 di un'inchiesta promossa dall'Institut für Sozialforschung di Francoforte e aggiornato sette anni dopo (anche con un capitolo dedicato alla Renault, a firma di Pierre Rolle) ${ }^{22}$. Pollock precisava poi:

Forse la cosa più opportuna è parlare di automazione soltanto nei casi in cui è data una delle cinque caratteristiche seguenti:

I. L'elaborazione di dati a mezzo di calcolatore elettronico (Electronic Data Processing, EDP).

2. Il controllo di interi processi di finitura o amministrativi a mezzo di calcolatori elettronici (Process Control Systems).

3. La 'ricerca operativa' (Operations Research).

4. Il 'controllo numerico' (numerical control) per il controllo immediato di macchineutensili.

19. V. Foa, La nuova linea nelle province, conclusioni del congresso provinciale della Fiom di Modena, 27 novembre 1955, ora in La cultura della Cgil. Scritti e interventi 1950-1970, Torino, Einaudi, 1984, p. 23; cfr. A. Ginzburg, Introduzione a V. Foa, Discorsi Parlamentari (1947-I988), Roma, Camera dei deputati, 20I4, pp. IX-LVII.

20. A. Graziani, Lo sviluppo dell'economia italiana. Dalla ricostruzione alla moneta europea, Torino, Bollati Boringhieri, 2000, pp. 65-69.

2I. D. Hounshell, Rethinking the Cold War; Rethinking Science and Technology in the Cold War; Rethinking the Social Study of Science and Technology, "Social Studies of Science», vol. 3I, n 2, aprile 200I, pp. 289-297.

22. F. Pollock, Automazione. Conseguenze economiche e sociali, Torino, Einaudi, I970 (I956), pp. 20-2I. 
5. Il concatenamento di macchine da lavoro a mezzo di impianti automatici transfer, facendo ricorso al principio della retroazione (Detroit Automation).

Tutte queste caratteristiche, e in particolare la quinta, andavano sviluppandosi alla Fiat e alla Renault sin dall'inizio degli anni I950, sotto lo sprone del Productivity Drive e della guerra di Corea. Nel dibattito sociologico sull'influsso delle nuove tecnologie sul lavoro, Georges Friedmann era una figura chiave, che tracciò di fatto le coordinate della disciplina in cui si mossero Alain Touraine, suo allievo, e Serge Mallet. Friedmann frequentava l'Italia, su invito di Adriano Olivetti tenne conferenze a Ivrea e pubblicò alcuni libri con le Edizioni Comunità: nel 1955 il più importante, Dove va il lavoro umano?, e nel 1963 il Trattato di Sociologia del lavoro scritto con Pierre Naville. È suo l'intervento di apertura di una sezione, intitolata Lavoratori e sindacati di fronte alle trasformazioni del processo produttivo, del convegno sul Progresso tecnologico e la società italiana organizzato da Franco Momigliano e Beniamino Andreatta ${ }^{23}$. A esso prendevano parte anche, tra gli altri, Gino Giugni, Luciano Gallino e Franco Ferrarotti della Olivetti, Foa, Trentin e Garavini della Fiom. Il convegno si tenne a Milano tra il 28 giugno e il 3 luglio 1960: per una suggestiva coincidenza, negli stessi giorni in cui s'incendiavano le manifestazioni di piazza contro l'apertura a destra del governo Tambroni.

Nel suo rapporto, scritto insieme al marxista belga Jacques Dofny, Friedmann ripercorreva le tappe dell'evoluzione dei processi produttivi dalla fine dell'Ottocento fino agli sviluppi dell'automazione. Egli la definiva secondo queste tre caratteristiche, in modo parzialmente differente da Pollock.

I. L'intégration, en une chaîne continue de production (que le produit parcourt sans être touché par la main de l'homme), de diverses opérations de fabrication jusqu'alors effectuées séparément;

2. Les dispositifs de rétroaction ou de "régulation automatique" munis de systèmes asservis (feedback ou closed loops) permettant l'ajustement du résultat effectif au résultat prévu; domaine de l'ingénieur électronicien, comme le sont aussi :

3. Les calculateurs et ordinateurs électroniques (computers) capables d'enregistrer et d'accumuler des informations, puis, sur la base de celles-ci, de faire des opérations

23. G. Friedmann e J. Dofny, Attitudes des travailleurs et position des organisations syndicales à l'egard du progrès technique (Rapport général d'introduction), in F. Momigliano (a cura di), Atti del Congresso internazionale di studio sul progresso tecnologico e la società italiana promosso dal Centro nazionale di prevenzione e difesa sociale e dal Comune di Milano sotto il patrocinio del Consiglio nazionale delle ricerche (Milano, 28 giugno-3 luglio 1960), vol. I: Lavoratori e sindacati di fronte alle trasformazioni del processo produttivo, Milano, Feltrinelli, I962, pp. $19-37$. 
mathématiques d'une complexité et avec une rapidité bien supérieures aux capacités du cerveau humain.

La vera e propria Detroit Automation consisteva nella prima di queste, della quale Friedmann prendeva ad esempio la Renault: l'automazione, proseguiva, imprime una drammatica accelerazione al progresso tecnico e dunque ai mutamenti nelle condizioni del lavoro e nella vita dei lavoratori ma anche, di conseguenza, all'apertura di nuove e vecchie contraddizioni tra la classe operaia e il capitale. L'aumento di potere formale delle organizzazioni operaie sia nella società che in seno alle singole aziende, dove esso si misurava nella capacità di interrompere il ciclo produttivo, nascondeva la crescente inadeguatezza del sindacato a comprendere e rispondere alla nuova situazione. La tendenza fondamentale dell'automazione era la riduzione della durata settimanale e giornaliera del lavoro: il tempo così liberato o consente di fare un secondo lavoro o è consacrato al consumo dei mass media. "Hors du lieu de travail, la consommation de mass media exerce une action dissolvante sur la "conscience de classe" ouvrière et, à travers elle, sur certains ressorts profonds du mouvement syndical». All'insufficienza del movimento operaio tradizionale di fronte alle sfide del neocapitalismo Friedmann dedicava le conclusioni della sua conferenza, che vale la pena riportare per intero.

En terminant cette esquisse, on peut se demander, sur la base des réflexions qui précèdent, si les syndicats, à travers la diversité de leurs structures, de leur orientation théorique et pratique, polarisés tantôt par une action para-politique, tantôt par l'organisation et les revendications professionnelles, sont adaptés à la civilisation technicienne et capables de réagir efficacement à ses transformations. Autrement dit, les réactions du mouvement syndical au progrès technique et particulièrement à l'automation sont conditionnées par son évolution depuis le début du siècle. En France, le syndicalisme de 1936 et plus encore de I960, si on le considère par-delà ses divisions, n'a que des rapports lointains avec le syndicalisme issu de la loi de I884: le nom subsiste, mais s'agit-il vraiment de la même institution? Une observation analogue ne simpose-t-elle pas pour l'Italie? Et que dire du rôle des organisations officielles dénommées "syndicats" dans les régimes à parti unique, où elles sont intégrées à l'État-patron, absorbées par lui et devenues essentiellement, entre ses mains, des instruments au service de la productivité?

En Occident et aux États-Unis, l'évolution du syndicalisme est inséparable de celle du capitalisme libéral vers un capitalisme interventionniste. Les transformations techniques à l'échelle de l'automation sinscriront nécessairement dans une économie de plus en plus dirigée dont le syndicalisme aspire, sous diverses formes, à contrôler la planification et la gestion. Est-il, dans sa phase actuelle, armé pour faire face à ces changements rapides dont nous avons marqué quelques-unes des incidences complexes, imprévues, sur les classes ouvrières? Enfin, les syndicats conservent-ils aujourd'hui, même dans les démocraties parlementaires, par rapport aux systèmes économiques et aux régimes politiques, une liberté d'action qui leur permette des réactions autonomes? 
À ces questions débordant le cadre qui nous a été fixé, seule l'histoire, et sans doute une histoire de longue durée, pourra répondre ${ }^{24}$.

È a un allievo di Friedmann, Alain Touraine, che si deve il più importante studio sulla Renault, pubblicato nel I955, frutto di una tesi di dottorato e di ricerche sul campo risalenti al 1948. Egli squarciava l'immagine pubblica di razionalismo monolitico e mostrava come all'interno della fabbrica, in virtù delle stesse dinamiche del progresso tecnico, si verificasse una sedimentazione e stratificazione di tecniche, tecnologie, modalità organizzative $^{25}$. Qui manca lo spazio per ricapitolare l'analisi delle strutture del lavoro d'industria, della disgregazione delle culture del mestiere, della subordinazione al sistema di macchine e alla determinazione dei tempi ${ }^{26}$.

Una prospettiva diversa animava le inchieste, poi raccolte nel 1963 sotto il titolo La nouvelle classe ouvrière ${ }^{27}$, che Serge Mallet svolgeva in tre fabbriche: le quattro sedi della Bull, all'avanguardia nella tecnologia delle schede perforate e quindi nell'informatizzazione del processo produttivo; la raffineria Caltex di Ambès nella Gironda; il ramo francese della compagnia elettrica Thomson-Houston a Bagneux. In tutto il libro è evidente non solo un circolo di militanza politica e scienza sociale, ma una vera esibizione muscolare della sociologia nella sua età dell'oro, una concezione volontaristica della scienza che nello studiare la società la voleva mutare ${ }^{28}$. Egli infatti, come il coetaneo François Furet, aveva lasciato il Pcf nel 1956 per fondare nel 1960 il Parti socialiste unifié, uno dei partiti più sensibili alle istanze dell'autogestione.

\footnotetext{
24. Ivi, pp. 36-37.

25. "Nous appelons Phase A, l'ancien système de travail, caractérisé par le travail qualifié de fabrication que requièrent les machines universelles ou "flexibles" [nella storia francese più o meno il periodo I8I5-1880], Phase B la période de transition, caractérisée par le développement du machinisme et du travail non qualifié d'alimentation des machines [1880-1930], Phase $\mathrm{C}$ la phase de l'automatisme et de l'élimination du travail directement "productif" [dal 1930 in poi]» (A. Touraine, L'Évolution du travail, cit., p. 48, corsivo dell'autore). La periodizzazione è presentata come schematica ed approssimativa a p. I75. A p. 139 una definizione del lavoro di catena: "Le travail à la chaîne est un type d'organisation du travail tel que les diverses opérations, réduites à une même durée ou à un multiple ou sous-multiple simple de cette durée, soient exécutées sans interruption entre elles et dans un ordre constant dans le temps et dans l'espace».

26. Touraine proponeva dunque una classificazione generale aggiornata, ammettendo che essa poteva solo fotografare un momento transeunte dell'evoluzione del lavoro: I. Manovale comune e manovale ai lavori pesanti; 2. Operaio su macchina (specializzato ordinario); 3. Operaio di fabbricazione (specializzato superiore); 4. Specializzato d'attrezzatura; 5. Specializzato ad alta qualificazione (cfr. ivi, p. I04).

27. S. Mallet, La nouvelle classe ouvrière, Parigi, Seuil, 1963.

28. "La théorie sociologique, à partir du moment où elle sort de son cercle d'auditeurs restreint pour attendre les larges masses par le truchement des hebdomadaires d'opinions, des revues, des militants, devient un élément constitutif de la vie sociale elle-même. Il arrive alors que la sociologie retrouve dans son interlocuteur le reflet de la théorie qu'il a forgée» (ivi, p. 25).
} 
L'adesione di Mallet allo strutturalismo, e quindi allo studio della fabbrica come una totalità interconnessa, non era priva di ambiguità. Essa portava alla ricerca di una contraddizione fondamentale identificata come agente della trasformazione ${ }^{29}$ — nella società industriale, la lotta di classe $\mathrm{e}$, infine, all'esaltazione della rivoluzione come rovesciamento della struttura. D'altra parte però, come notava Liliana Lanzardo in una recensione sui Quaderni Rossi ${ }^{30}$, rischiava spesso di considerare non solo il progresso tecnologico come una forza di per sé neutrale, ma anche la presa di coscienza da parte dei lavoratori delle proprie capacità tecniche come un fatto automatico, "predeterminato dalla struttura». Una versione sovversiva dello strutturalismo, insomma, in cui lo scienziato sociale diventava il polo volontaristico e la società quello deterministico.

Per Mallet la crescente integrazione degli operai nella struttura era il riflesso dei mutamenti nella produzione: le riforme a livello di salario, di sicurezza dell'impiego e di formazione professionale, messe in atto a livello aziendale o a livello statale, erano qualitativamente diverse dalle antiche forme di paternalismo padronale ${ }^{31}$; e a questo si doveva sia l'isolamento dell'operaio in fabbrica, ovvero il suo rapporto alienato con la macchina ("Plus nombreux est le collectif ouvrier, plus grand est l'isolement de chaque ouvrier à sa machine»), sia la completa burocratizzazione, in sostanza la sclerotizzazione del sindacato, sempre più coinvolto nella cogestione delle relazioni industriali. Sia la Cgt che la Cgil avevano di fatto accettato le coordinate della cosiddetta "participation conflictuelle», ovvero della collaborazione aziendale ${ }^{32}$.

Torniamo così al punto dolente dell'insufficienza delle sinistre di fronte alle sfide poste dal neocapitalismo. Rimangono esemplari di questo momento, nel dibattito italiano ma anche nella sua dimensione europea e mondiale, i convegni economici organizzati dall'Istituto Gramsci, nei quali gli intellettuali socialisti e comunisti tentavano di comprendere le nuove forme assunte del capitalismo, orientandosi più che a divinare il suo destino - superamento delle contraddizioni o tradizionale ipotesi crollista - a individuarne le tendenze oggettive e le possibilità aperte: mi riferisco a Tendenze del capitalismo italiano (1962), dove Trentin teneva la celebre relazione sulle ideologie del neocapitalismo e Lucio Magri l'intervento, poi ripreso da Les Temps modernes, in cui affermava (contro un consistente filone

29. "La notion de contradiction, chez Marx comme chez Hegel, est une notion structuraliste» (ivi, p. I7).

30. L. Lanzardo, Recensione, "Quaderni Rossi», nº 5, aprile 1965, pp. I73-183.

3I. S. Mallet, La nouvelle classe ouvrière, cit., p. 54.

32. Ivi, p. 44. 
della tradizione del Pci, portato avanti soprattutto da Giorgio Amendola) che l'Italia non poteva più essere considerata un paese arretrato, bensì soffriva ormai di tutte le contraddizioni del capitalismo avanzato ${ }^{33}$; e a Tendenze del capitalismo europeo (1965), cui prendevano parte anche André Gorz, André Barjonet, Charles Bettelheim ${ }^{34}$.

Uno dei protagonisti di questo secondo convegno era Lelio Basso, con una relazione sulle Prospettive della sinistra europea ${ }^{35}$. Questi, come si è già visto, ribadiva che «la società capitalistica rimane essenzialmente una società conflittuale, ma possiede strumenti che possono attenuare i contrasti e creare forme di consenso e di integrazione»: anzi, se il benessere fordista era un fattore oggettivo, la socialdemocrazia riformista era uno dei più importanti fattori politici. Un'autentica strategia marxista sarebbe stata, allora, quella che discendeva da un'analisi coraggiosa tanto delle novità quanto delle persistenti contraddizioni del neocapitalismo. Su tale base dunque, mentre maturava un crescente interesse per i movimenti rivoluzionari in Asia e in America Latina, Basso invitava il movimento operaio europeo a rinunciare all'idea di prendere il potere impadronendosi dei centri decisionali centrali — le 'stanze dei bottoni', nell'infelice terminologia di Nenni - fossero le istituzioni parlamentari o gli organismi tecnici di un apparato statale neutrale; si trattava, in sostanza, di mettere in pratica una lotta "per attaccare il potere privato, per affermare ovunque e realizzare progressivamente il principio che le decisioni di interesse collettivo [...] devono essere decisioni democratiche degli interessati che costituiscono la grande massa dei lavoratori» ${ }^{36}$.

L'orizzonte di Lelio Basso, in sintesi, era quello di una democrazia industriale intesa come risposta politica della classe operaia al carattere sociale raggiunto dalla produzione capitalistica, alla concentrazione del potere in mani private. Nonostante riconoscesse che il potere era ormai diffuso, non centralizzato, la prospettiva di Lelio Basso rimaneva però focalizzata 'in alto', alla conquista dello Stato; quella di Vittorio Foa, in tutti i suoi scritti fino agli anni 1980, andava 'in basso', riportava l'attenzione alla quotidianità della fabbrica, alla politicità del lavoro.

33. Tendenze del capitalismo italiano. Atti del convegno economico dell'Istituto Gramsci (Roma, 23-25 marzo 1962), Roma, Editori Riuniti, I962; cfr. anche Lucio Magri, Le modèle de développement capitaliste et le problème de l'alternative prolétarienne, "Les Temps modernes», n 196-197, settembre-ottobre 1962.

34. Tendenze del capitalismo europeo. Atti del convegno dell'Istituto Gramsci (Roma, 25-27 giugno I965), Roma, Editori Riuniti, 1966.

35. L. Basso, Le prospettive della sinistra europea, in Tendenze del capitalismo europeo, cit., pp. 253-308.

36. Id., Neocapitalismo e 'socialisti moderni', cit., p. 28I. 
La prospettiva rivoluzionaria per la realizzazione del socialismo, cioè per il controllo collettivo delle forze di produzione, ha il suo centro nella struttura, cioè nella lotta quotidiana, concreta, anche graduale, per accrescere il controllo popolare e pubblico, per limitare e liquidare il controllo irresponsabile dei gruppi sulle gigantesche forze nuove entrate in azione per lo sviluppo economico, e per quelle ancora più grandi che la nuova scienza e la nuova tecnica stanno mettendo a disposizione dell'umanità.

Per chiudere con questo bel brano di Foa faccio un salto indietro di qualche anno. Esso infatti proviene da un intervento del 1957, cui Panzieri su «Mondo Operaio» dava il titolo Il neocapitalismo è una realtà. In questo articolo Foa rifiutava con nettezza la «nuova ondata 'conciliatrice' tra capitale e lavoro» contrabbandata sotto l'etichetta del neocapitalismo, ma sottolineava anche che sotto questo nome si cercava di descrivere cambiamenti profondi, oggettivi, nella struttura del capitalismo. Cambiamenti che mettevano in crisi le tradizioni di lotta e di teorie delle sinistre, ai quali forse, come diceva Friedmann, potrà rispondere solo la storia — anzi «une histoire de longue durée». Per scoprire la crisi, in sostanza, il marxismo e il movimento operaio non possono aspettare l'ora del tracollo: devono essere in grado d'individuarne i contorni già nei tempi di vacche grasse.

Chi oggi cercasse ancora la crisi nel ristagno e nell'immobilità, e non invece, com'è giusto, nei modi e nelle forme, nuovi e diversi gli uni dalle altre, delle forze produttive messe in moto dalla tecnica e dalla scienza, finirebbe con l'essere travolto da queste stesse forze produttive e lasciare campo libero al potere del nuovo nemico ${ }^{37}$.

37. V. Foa, Il neocapitalismo è una realtà, ora in La cultura della Cgil, cit., pp. 4I-42. 